\title{
Control of Neisseria gonorrhoeae pilin gene expression by environmental factors: involvement of the pilA/pilB regulatory genes
}

\author{
Mireille Larribe, ${ }^{1}$ Muhamed-Kheir Taha, ${ }^{1}$ Andrzej Topilko ${ }^{2}$ \\ and Christian Marchal' \\ Author for correspondence: Mireille Larribe. Tel: +331456889 58. Fax: + 33145688953. \\ e-mail: mlarribe@pasteur.fr
}

Unité des Neisseria ${ }^{1}$ and Station Centrale de Microscopie Electronique? Institut Pasteur, 28 rue du Dr Roux, 75724 Paris Cedex 15, France

\begin{abstract}
The control of the expression of the pilin gene (pilE) in Neisseria gonorrhoeae under a wide variety of growth conditions has been studied. The expression of pile was measured using transcriptional fusions between pilE and the gene encoding chloramphenicol acetyltransferase (CAT), and the level of pilin production was measured by Western blot analysis. Many of the conditions tested affected both growth rate and pilin gene expression (e.g. isoleucine, high osmolarity, high temperature, anaerobic growth, pH 6, urea and iron depletion). Changes in the level of many other proteins were also observed, depending on the conditions, indicating that gonococci undergo an adaptive response to environmental variations. Moreover, environment-induced changes in the level of many proteins, including pilin, seem to involve the pilA/pilB regulatory system, which has been previously proposed to modulate the expression of the gonococcal pilin gene.
\end{abstract}

Keywords: Neisseria gonorrhoeae, pilin expression, environmental factors

\section{INTRODUCTION}

Neisseria gonorrhoeae is an exclusively human pathogen which colonizes different mucosal surfaces of the urogenital tract and other sites such as the rectum, the oropharynx or the cornea (Watt \& Ward, 1980; Gorby \& Schaefer, 1992; Molesh et al., 1994). The bacteria are exposed to a variety of different growth conditions during infection. The ability of the gonococcus to infect its host is conditioned by successive steps of interactions with host cells and by the survival of the bacteria.

Gonococcal pili, which are composed mainly of subunits called pilin, are required for initial attachment of the bacteria to epithelial cells (Swanson et al., 1987), probably in conjunction with outer-membrane proteins such as PilC (Rudel et al., 1995). Opa proteins, which are localized in the outer membrane, are also involved in cell-bacteria interactions (Waldbeser et al., 1994). Pilin and Opa proteins undergo phase and antigenic variation (Segal et al., 1985; Seifert \& So, 1988; Robertson \& Meyer, 1992). In addition, the pilin gene is transcriptionally regulated by the products of two genes, pilA/pilB, which act in a manner analogous to the

Abbreviation: CAT, chloramphenicol acetyltransferase. family of bacterial two-component regulators (Taha et al., 1988, 1991). The pilA gene seems to be essential and its product appears to play a crucial role related to bacterial cell growth. Indeed, when pilA is inactivated by transposon insertion, only the pilA $A^{+} /$pilA:: $\mathrm{mTn} 3 \mathrm{Cm}-3$ heterodiploids are viable (Taha et al., 1988). In addition, it has been shown that PilA protein is implicated in the gonococcal stress response (Taha et al., 1992) and in the resistance to the bactericidal effect of normal human serum (Taha, 1993). The cellular location of PilA and PilB, the similarities in amino acid sequence with members of the family of twocomponent regulators and the phosphorylation of PilA (Taha \& Giorgini, 1995) suggest that these two proteins may be involved in the modulation of pilin gene expression by environmental factors.

In the present study, we investigated the effects of numerous growth conditions on the control of expression of the pilin gene, some of which reflect the habitat of N. gonorrboeae (high osmolarity, high urea concentration, anaerobiosis). The level of regulation of pilin gene expression was analysed using a transcriptional fusion between the cat gene [encoding chloramphenicol acetyltransferase (CAT)] and the promoter of the pilin gene (pilE) in N. gonorrhoeae strain MS11. The influence of growth conditions on pilE transcription 
and on the amount of pilin synthesized is reported. The role of the pilA/pilB system in the response to these external signals was also analysed using $p i l A$ and $p i l B$ mutants. Variations in the amounts of several proteins, such as the heat-shock protein Gsp63, under these different conditions were also tested. We discuss the role of PilA and PilB in gonococcal adaptation to the environment.

\section{METHODS}

Bacterial strains and growth conditions. The variants of the gonococcal strain MS11 used in this study are listed in Table 1. The transcriptional fusions between the pilE promoter and the cat gene at the pilE1 site (CAT-1) or pilE2 site (CAT-2) of the strain MS11 have been previously described (Taha et al., 1988). The strains harbouring the pilE-cat fusions produce CAT as well as the pilin protein. The mutations in pilA, pilB and opaE1 obtained by insertions of the $m \operatorname{mn} 3 \mathrm{Cm}-3$ transposon were previously described by Taha et al. (1988). The pilA mutants are always heterodiploid for the pilA gene and homodiploid for the neighbouring gene, pilB. The mutation pilA $k$ results from the insertion of a $B a m H I$ fragment carrying the $a p h-3^{\prime}$ gene of Tn5 (encoding the aminophosphotransferase and conferring kanamycin resistance) into the NotI site in pilA (Taha \& Giorgini, 1995). Bacteria were grown at $37{ }^{\circ} \mathrm{C}$ under $5 \%(\mathrm{v} / \mathrm{v}) \mathrm{CO}_{2}$ on $\mathrm{G}$ medium with $\mathrm{G}$ supplements (Sanofi-Diagnostics Pasteur) or on the minimal medium as previously described by Larribe et al. (1991). These conditions are indicated as standard growth conditions. When needed, chloramphenicol and kanamycin were added at final concentrations of $10 \mu \mathrm{g} \mathrm{ml}^{-1}$ and $40 \mu \mathrm{g} \mathrm{ml}^{-1}$, respectively. The effect of amino acid supplements was studied on solid minimal or $\mathrm{G}$ medium. For anaerobic cultures, the Petri dishes containing sodium nitrite discs $(40 \mathrm{mM})$ were incubated in an anaerobic chamber under $\mathrm{CO}_{2} / \mathrm{H}_{2}(5: 95, \mathrm{v} / \mathrm{v})$. The studies of increased osmolarity were performed in liquid minimal or $\mathrm{G}$ medium. Iron starvation was achieved in a solid minimal medium in which iron had been chelated by the siderophore desferrioxamine (Desferal; $15 \mathrm{mM}$ ). Growth at $41^{\circ} \mathrm{C}$ was performed on solid $G$ medium.

CAT assay. The cells were centrifuged, washed, resuspended in

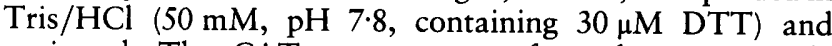
sonicated. The CAT assay was performed as previously described by Shaw (1975). CAT activity is expressed as $\mathrm{U}$ (mg protein $)^{-1}$ in total cell extracts (1 CAT unit corresponding to $1 \mathrm{nmol}$ chloramphenicol acetylated $\left.\mathrm{min}^{-1}\right)$. The results obtained under a given growth condition were expressed as the percentage of the value measured under standard growth conditions. Experiments were repeated at least twice and usually about five times.

The CAT assay cannot be used to monitor pilE expression in the pilA/pilB mutants since their mutations were obtained by the insertion of the transposon $\min 3 \mathrm{Cm}-3$, which confers chloramphenicol resistance. In order to study the role of PilA and PilB in the modulation of pilE gene expression under different environmental conditions, the amounts of pilin were measured by Western blot analysis. In each case, the results are expressed as the percentage of the pilin level in the wildtype strain MS11-A.

Pilin detection by Western blot analysis. Western blotting was carried out using ${ }^{35} \mathrm{~S}$-labelled protein A (Amersham) as previously described by Dupuy et al. (1991). The anti-pilin serum was prepared as previously described by Dupuy et al. (1991). After exposure, the bands were cut out of the membrane and radioactivity was measured in a liquid scintillation counter. Results were expressed as the percentage of c.p.m. (mg total protein $)^{-1}$ for a given growth condition compared to the standard growth conditions. Experiments were performed at least twice and generally five times. SDSPAGE analyses were performed for protein profiles; the gels were stained with Coomassie Brilliant Blue or with silver nitrate.

Electron microscopy. Gonococci from solid or liquid cultures were gently dissociated and resuspended in phosphate buffer. Copper grids (400 mesh) coated with collodion-carbon support film were floated for $3 \mathrm{~min}$ on the bacterial suspension. For rotary shadowing, the grids with attached gonococci were mounted on a rotary holder. The stage was positioned $10 \mathrm{~cm}$ from a platinum-carbon source and adjusted to an angle of $6^{\circ}$ with respect to the electron gun. Samples were rotary-shadowed (100 r.p.m.). Metal shadowing was sufficient after $20 \mathrm{~s}$ evaporation. All specimens were examined using a JEOL JEM100CXII electron microscope.

\section{RESULTS}

\section{Pilin gene expression is related to growth rate in N. gonorrhoeae}

We first tested the effect of environmental factors on growth of $N$. gonorrboeae on standard G and minimal media. Growth was evaluated by assaying the protein content of cells from solid or liquid medium cultures after the same incubation time. Several factors reduced growth moderately $(50 \%$ reduction of growth under

Table 1. Bacterial strains

\begin{tabular}{|c|c|c|}
\hline Strain & Genotype or description & Reference \\
\hline MS11-A & pilE1, pilE2 & Segal et al. (1985) \\
\hline $4.1 \mathrm{C}$ & pilE1, $\Delta$ pilE2, derived from MS11-A & Segal et al. (1985) \\
\hline MS11.opaE1a & opaE1:: $\operatorname{mTn} 3 \mathrm{Cm}-3$ & Taha et al. (1988) \\
\hline MS11.CAT-1 & pilE1-cat (transcriptional fusion) & Taha et al. (1988) \\
\hline MS11.CAT-2 & pilE2-cat (transcriptional fusion) & Taha et al. (1988) \\
\hline MS11.pilBa & pilB:: $\mathrm{mTn} 3 \mathrm{Cm}-3$ & Taha et al. (1988) \\
\hline MS11.pilA ${ }^{+} / A a$ & pilA:: $\mathrm{mTn} 3 \mathrm{Cm}-3$ & Taha et al. (1988) \\
\hline MS11.pilA $A^{+} / A k$ & pilA::aph-3' & This work \\
\hline MS11.pilA ${ }^{+} / A k . p i l B a$ & pilA::aph-3'; pilB:: mTn $3 C m-3$ & This work \\
\hline
\end{tabular}


Table 2. CAT activity and level of pilin of the different strains under standard growth conditions

The standard minimal medium contained $30 \mathrm{mM}$ dextrose, $4 \mathrm{mM} \mathrm{NH}_{4} \mathrm{Cl}, 0.35 \mathrm{mM}$ cysteine, $0.15 \mathrm{mM}$ cystine, $39 \mathrm{mM} \mathrm{P}$, $0.25 \mathrm{mM} \mathrm{CaCl}_{2}$ and $2 \mathrm{mM} \mathrm{MgCl}_{2}$ and was incubated at $37^{\circ} \mathrm{C}$ under $5 \% \mathrm{CO}_{2}$.

\begin{tabular}{|lcc|}
\hline Strain & CAT activity* & Pilin $\dagger$ \\
\hline MS11.CAT-1 & $100(430 \pm 50)$ & $92 \pm 4$ \\
MS11.CAT-2 & $100(310 \pm 37)$ & $78 \pm 3$ \\
MS11-A & 100 \\
MS11.pilBa & $125 \pm 33$ \\
MS11.pilA ${ }^{+} /$pilAa & $40 \pm 8$ \\
MS11.pilA $/$ pilAk & $140 \pm 2$ \\
MS11.pilA $/$ pilAk.pilBa & $140 \pm 2$ \\
\hline
\end{tabular}

*For MS11.CAT-1 and MS11.CAT-2, CAT activity under standard conditions was taken as $100 \%$. The absolute values of CAT activity for each fusion are indicated in parentheses and expressed as $U$ (mg protein $)^{-1} \pm \mathrm{sD}$. One CAT unit $=1 \mathrm{nmol}$ chloramphenicol acetylated $\mathrm{min}^{-1}$ at room temperature.

†For each strain under standard growth conditions, the level of pilin is expressed as the percentage $\pm S D$ of values obtained with MS11-A wild-type.

anaerobic conditions and temperature between 30 and $\left.37^{\circ} \mathrm{C}\right)$. Other factors reduced growth severely $(90 \%$ reduction of growth) [high osmolarity $(300 \mathrm{mM} \mathrm{NaCl}$ or $\mathrm{KCl}$, or $600 \mathrm{mM}$ sucrose), iron depletion, $400 \mathrm{mM}$ urea, increased temperature from 37 to $41^{\circ} \mathrm{C}$ and $\mathrm{pH} 6$. These results revealed a very narrow range of favourable growth conditions, especially for temperature, $\mathrm{pH}$, saline concentration and oxygenation.
The virulence and the piliation of the gonococcus have been previously reported to be modified in the presence of amino acids (Saikh et al., 1989), during anaerobiosis or under limitation for glucose, cystine or iron (Keevil et al., 1986, 1989). We analysed the effects of environmental factors on the transcription of the pilE1 and pilE2 genes by using a transcriptional fusion between the pilin gene promoter and the promoter-less cat gene encoding CAT (Taha et al., 1988). The pilE-cat fusion was integrated by homologous recombination into the wild-type strain MS11-A at the pilE1 site (CAT-1) or at the pilE2 site (CAT-2). The pilin level was also evaluated by Western blot analysis using anti-pilin antibodies. The CAT activities obtained with the two fusions CAT-1 and CAT-2 (Table 2) showed expression at both sites. However CAT-2 showed a reproducibly lower level of expression than CAT-1. Pilin level was not affected by the insertion of the cat gene (Table 2). As indicated in Table 3, all conditions which reduced the gonococcal growth rate in the wild-type MS11-A strain were also shown to reduce pilin gene transcription and pilin level, with the exception of iron depletion. Indeed, the latter condition, which severely reduced the growth rate, seemed to have no effect on pilin gene transcription or on the pilin level (Table 3).

The presence of some amino acids seemed to affect growth rate and pilin gene expression in the same manner. When isoleucine was added at $3 \mathrm{mM}$, it induced a fourfold and twofold increase in the expression of the pilE-cat fusion and the pilin level, respectively (Table 3). It also provoked a threefold increase in growth rate.

We next examined the piliation of wild-type strain MS11-A under standard and high osmolarity growth conditions using electron microscopy. We observed that

Table 3. CAT activity and level of pilin under different environmental conditions

CAT activity and level of pilin are expressed as the percentage $\pm S D$ of the values observed in standard G medium.

\begin{tabular}{|c|c|c|c|c|c|c|c|c|}
\hline \multirow[t]{2}{*}{ Strain } & \multirow[t]{2}{*}{ pH 6} & \multirow[t]{2}{*}{ Anaerobiosis } & \multirow[t]{2}{*}{$41^{\circ} \mathrm{C}$} & \multicolumn{2}{|c|}{ High osmolarity ${ }^{*}$} & \multirow{2}{*}{$\begin{array}{c}\text { Urea } \\
(400 \mathrm{mM})\end{array}$} & \multirow{2}{*}{$\begin{array}{l}\text { Isoleucine } \\
(3 \mathrm{mM})\end{array}$} & \multirow{2}{*}{$\begin{array}{c}\mathrm{Fe} \\
\text { starvation }\end{array}$} \\
\hline & & & & $\mathrm{KCl}$ & $\mathrm{NaCl}$ & & & \\
\hline \multicolumn{9}{|l|}{ MS11.CAT-1 } \\
\hline CAT activity & $52 \pm 9$ & $28 \pm 7$ & $65 \pm 7$ & $41 \pm 8$ & $39 \pm 7$ & $22 \pm 11$ & $558 \pm 180$ & $162 \pm 23$ \\
\hline Pilin & $48 \pm 28$ & $34 \pm 8$ & $51 \pm 3$ & $51 \pm 2$ & $56 \pm 2$ & $51 \pm 2$ & $242 \pm 3$ & $124 \pm 8$ \\
\hline \multicolumn{9}{|l|}{ MS11.CAT-2 } \\
\hline CAT activity & $37 \pm 20$ & $37 \pm 7$ & $49 \pm 7$ & $39 \pm 7$ & $34 \pm 11$ & $19 \pm 8$ & $640 \pm 250$ & $128 \pm 15$ \\
\hline Pilin & $52 \pm 12$ & $30 \pm 6$ & $58 \pm 17$ & $77 \pm 16$ & $69 \pm 7$ & $63 \pm 6$ & $309 \pm 137$ & $145 \pm 7$ \\
\hline \multicolumn{9}{|l|}{ MS11 } \\
\hline Pilin & $43 \pm 6$ & $47 \pm 8$ & $39 \pm 4$ & $83 \pm 10$ & $69 \pm 15$ & $85 \pm 15$ & $170 \pm 6$ & $148 \pm 13$ \\
\hline \multicolumn{9}{|l|}{ MS11.pilBa } \\
\hline Pilin & $156 \pm 40$ & $85 \pm 31$ & $73 \pm 50$ & $168 \pm 17$ & $270 \pm 40$ & $159 \pm 17$ & $77 \pm 3$ & $84 \pm 10$ \\
\hline \multicolumn{9}{|l|}{ MS11.pilA $A^{+} /$pilAa } \\
\hline Pilin & $76 \pm 11$ & $12 \pm 5$ & $47 \pm 14$ & & & $97 \pm 18$ & $42 \pm 3$ & $185 \pm 35$ \\
\hline \multicolumn{9}{|l|}{ MS11.pilA $A^{+} /$pilAk } \\
\hline Pilin & $75 \pm 13$ & $82 \pm 15$ & $96 \pm 13$ & & & $102 \pm 6$ & $156 \pm 50$ & $120 \pm 10$ \\
\hline
\end{tabular}

"High osmolarity was obtained by addition of $300 \mathrm{mM} \mathrm{KCl}$ or $\mathrm{NaCl}$. 

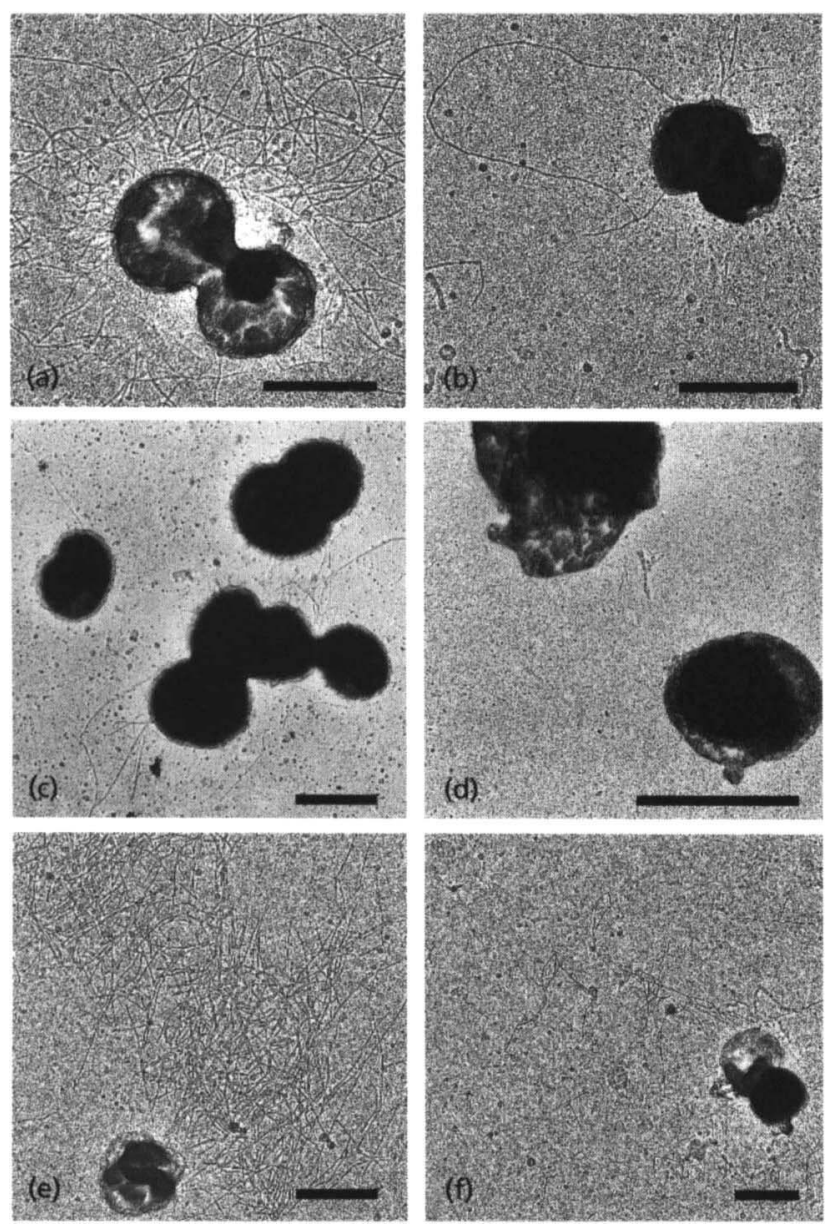

Fig. 1. Piliation status of wild-type gonococci and mutants observed by electron microscopy using the rotative shadowing method. Two growth conditions were tested: minimal medium $(\mathrm{a}, \mathrm{c}, \mathrm{e})$ and minimal medium supplemented with $300 \mathrm{mM} \mathrm{KCl}$ (b, d, f). (a, b) MS11-A wild-type strain; (c, d) 4.1C wild-type strain; (e, f) mutant MS11.pilBa. Bars, $1 \mu \mathrm{m}$.

the bacteria grown under high osmolarity were almost devoid of pili, although the pilin level was only reduced by $50 \%$ in strain MS11-A (Fig. 1).

Taken together, these data indicate that pilin gene expression and growth rate are clearly related and that $N$. gonorrhoeae modulates the expression of the pilin gene in response to external signals.

\section{Analysis of pilA and pilB mutants}

We next analysed the behaviour of pilA and pilB mutants under different growth conditions described in this study. We tested the previously described $p i l A^{+} / p i l A a$ heterodiploid and pilBa mutant (Taha et al., 1988). Moreover, we constructed a new pilA heterodiploid (pilA ${ }^{+} /$pilAk). The pilAk allele was obtained by the insertion of the $a p h-3^{\prime}$ gene (which confers resistance to kanamycin) into the NotI site in the pilA gene (Fig. 2). The new recombinant plasmid, pNG17K, was then used to transform the wild-type strain MS11-A and the mutant MS11.pilBa. All pilA mutants were heterodiploid pilA ${ }^{+} /$pilA:: $\mathrm{mTn} 3 \mathrm{Cm}-3$ or pilA ${ }^{+} /$pilA::aph-3' (data not shown). In the gonococcus, it has been previously shown that pilA is an essential gene, as its inactivation by transposon insertion is lethal, and only pilA $A^{+}$pilA : : m Tn $3 \mathrm{Cm}-3$ heterodiploids can be obtained (Taha et al., 1988). In the pil $A^{+} /$pilAa heterodiploid, a reduction in pilin gene transcription is observed. This phenotype results from the negative transdominant effect of the product of the pilAa allele on that of the wild-type allele (Taha et al., 1988). Moreover, the phenotype of pilA heterodiploids depends on the site of the insertional inactivation on the pilA gene (Taha et al., 1991).

Under standard growth conditions (minimal medium), the amount of pilin was higher in the mutants $p i l B a$, $p i l A^{+} / p i l A k$ and $p i l A^{+} / p i l A k$.pilBa when compared to the strains MS11-A, MS11.CAT-1 and MS11.CAT-2. The pilin level was lower in the mutant $p_{i l A^{+} / p i l A a}$ (Table 2; Fig. 3, lanes 6-10).

The external conditions tested in this study which modified the amount of pilin had few or no effects in the pilBa mutant (Table 3; Fig. 3, lanes 3 and 8), in particular $\mathrm{pH} 6$, urea (which reduced the pilin level in the wild-type strain) and isoleucine (which increased the pilin level in the wild-type strain). However, the piliation state of the pilBa mutant is severely reduced under high osmolarity conditions (Fig. 1e, f). These data indicate that factors other than the pilin level are also involved in piliation in the gonococci. The pilA ${ }^{+} / p i l A k$ heterodiploid showed the same behaviour as the pilBa mutant (Table 3; Fig. 3, lanes 4 and 9). A different situation was observed for the heterodiploid $p i l A^{+} / p i l A a$. Indeed, the effect of growth at $\mathrm{pH} 6$ or in the presence of $400 \mathrm{mM}$ urea on pilin level was abolished in this heterodiploid (Table 3). The presence of $3 \mathrm{mM}$ isoleucine reduced the pilin level in the $p i l A^{+} / p i l A a$ heterodiploid while it increased this level in the wild-type strain (Table 3). However, other conditions, growth under anaerobiosis or at $41^{\circ} \mathrm{C}$ (Fig. 3 , compare lanes 1 and 2 to 6 and 7), had the same effect on both the wild-type strain and the pilA $A^{+} / p i l A a$ heterodiploid. The modulation of pilin synthesis seemed to be more pronounced in the $p i l A^{+} / p i l A a$ heterodiploid for the growth under anaerobic conditions (Table 3). These data indicate that the response of pilE gene expression in N. gonorrhoeae to several external signals passes through the pilA/pilB regulatory system.

\section{Analysis of the protein profile in pilA/pilB mutants compared to the wild-type strain}

It has been suggested previously that pilA/pilB genes constitute a pleiotropic regulatory system which could be involved in the regulation of several aspects of the gonococcal virulence (Taha et al., 1992; Taha, 1993). In view of this, we examined the pleiotropic effects of pilA/pilB mutations on the level of other proteins produced by $N$. gonorrhoeae.

We first used SDS-PAGE analysis of total cell proteins to compare the protein profile of the wild-type strain 


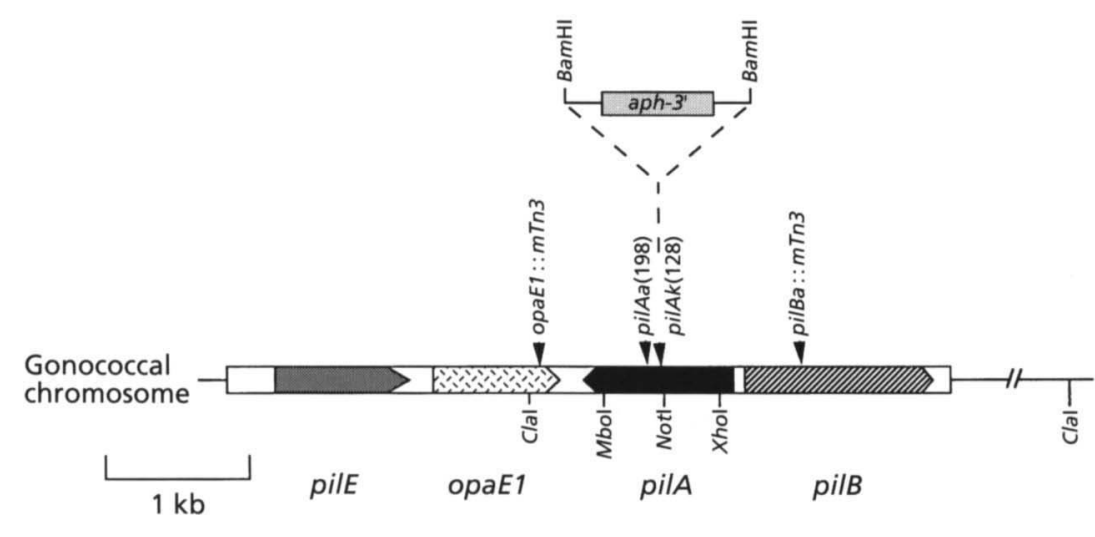

Fig. 2. Schematic representation of the pilA/pilB region of $N$. gonorrhoeae. Black arrowheads indicate the position of the mutational insertions of $\mathrm{mTn} 3 \mathrm{Cm}-3$ or aph-3', with numbers in parentheses corresponding to the amino acid deduced from the nucleotide sequence.

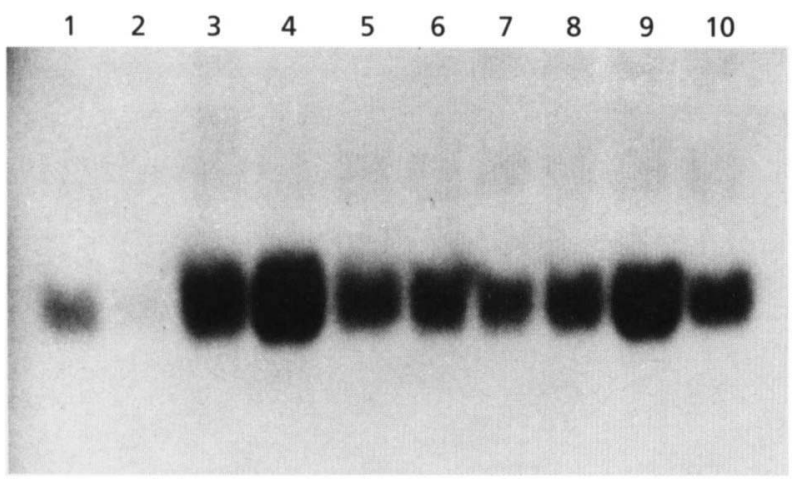

Fig. 3. Western blot analysis of whole-cell extracts of $N$. gonorrhoeae using anti-pilin antibody. Bacteria were grown at $41^{\circ} \mathrm{C}$ (lanes 1-5) or at $37^{\circ} \mathrm{C}$ (lanes $6-10$ ). The strains analysed were MS11-A (lanes 1 and 6), MS11.pilA+/pilAa (lanes 2 and 7), MS11.pilBa (lanes 3 and 8), MS11.pilA $A^{+} /$pilAk (lanes 4 and 9) and MS11.pilA ${ }^{+} /$pilAk.pilBa (lanes 5 and 10).

MS11-A under the standard growth conditions to those under anaerobic, high temperature or high osmolarity growth conditions. The levels of several proteins were observed to be modified in the wild-type strain grown under the conditions tested compared to the standard growth conditions. These data are summarized in Table 4. Variations in the amounts of some proteins were specific for one condition. Some other proteins were modulated in the same way under the three conditions tested (e.g. the $66 \mathrm{kDa}$ protein).

We observed differences between pilA or pilB mutants and the wild-type strain MS11-A grown under standard conditions. As shown in Table 4, each mutant $\left(p i l A^{+} / p i l A a\right.$, pilA $A^{+} / p i l A k$ and pilBa) has a distinct protein profile. Indeed, the increase in the $66 \mathrm{kDa}$ and the $41 \mathrm{kDa}$ proteins observed in the wild-type strain under anaerobic, high temperature or high osmolarity growth conditions is also observed in the pilA $A^{+} / p i l A a$ mutant grown under standard growth conditions. In the same manner, the increase of proteins of 170,66 and $53 \mathrm{kDa}$ observed under high osmolarity or under anaerobic growth conditions in the wild-type strain is also present in the pilBa mutant grown under standard conditions. These data indicate that the modulation observed in the level of some proteins in the mutants mimics that observed in the wild-type strain under these growth conditions.

We also compared the ability of the wild-type strain and the different mutants to adapt their protein level when shifted to different growth conditions. The pilAk mutation abolishes or reduces the ability to modulate production of various proteins according to environmental conditions, e.g. under anaerobiosis (Fig. 4, compare lane 3 to lane 7 ) or at $41^{\circ} \mathrm{C}$ or under high osmolarity (data not shown). The mutation pilBa affects the changes in protein profile at least under high osmolarity (Larribe et al., 1991) and acidic conditions (data not shown). The pilA $A^{+} /$pilAa mutant conserved the ability to change the relative amount of different proteins at least after the switch to anaerobiosis (Fig. 4, compare lane 1 to lane 10).

As the growth conditions tested in this study may be viewed as stress conditions, we next analysed the level of the gonococcal protein Gsp63, which is related to the GroEL protein of Escherichia coli. Gsp63 has been reported to be increased in gonococci grown under stress conditions (Pannekoek et al., 1992). We used monoclonal antibodies directed against epitope G6 of the Gsp63 protein to measure the level of this protein (Pannekoek et al., 1992). When compared to the wildtype strain, the level of Gsp63 was increased under standard growth conditions in the pilA $A^{+} /$pilAa heterodiploid, confirming previous results (Fig. 5) (Taha et al., 1992). Under growth at $41^{\circ} \mathrm{C}$, the pilBa mutant and the $p i l A^{+} / p i l A k$ heterodiploid showed an increase in the level of Gsp63, while the $p i l A^{+} / p i l A a$ heterodiploid showed a decrease in the level of this protein (Fig. 5). Surprisingly, the level of Gsp63 decreased in the wild-type strain and in the pilA $A^{+} /$il Aa heterodiploid mutant under anaerobic growth conditions while it increased in $p i l A^{+} / p i l A k$ and pil $A^{+} /$pilAk.pilBa mutants. These data indicate that gonococci undergo an adaptive response upon exposure to stress conditions and that the regulatory system pilA/pilB seems to be involved in the induction of this response. 
Table 4. SDS-PAGE analysis of total proteins of cells grown in different modified media compared to standard medium

\begin{tabular}{|c|c|c|c|c|c|c|}
\hline \multirow{2}{*}{$\begin{array}{l}\text { Mol. mass } \\
(\mathrm{kDa})\end{array}$} & \multicolumn{3}{|c|}{ Wild-type MS11* } & \multicolumn{3}{|c|}{$\begin{array}{l}\text { MS11 mutants under } \\
\text { standard growth conditions } \dagger\end{array}$} \\
\hline & Osmolarity & $41^{\circ} \mathrm{C}$ & Anaerobiosis & $\overline{p i l A^{+} / A a}$ & pil $A^{+} / A k$ & $p i l B a$ \\
\hline 180 & & + & & + & & \\
\hline 170 & + & & + & & - & + \\
\hline 150 & & & - & & & \\
\hline 120 & + & & + & & & \\
\hline 100 & + & & & & & + \\
\hline 97 & & - & - & & & \\
\hline 95 & & - & & & & \\
\hline 90 & - & - & - & & & \\
\hline 83 & + & & + & & & \\
\hline 80 & & - & & & + & \\
\hline 73 & - & - & - & + & $\begin{array}{l}+ \\
-\end{array}$ & - \\
\hline 66 & $\begin{array}{l}+ \\
-\end{array}$ & $\begin{array}{l}+ \\
-\end{array}$ & + & $\begin{array}{l}+ \\
+\end{array}$ & $\begin{array}{l}- \\
+\end{array}$ & $\begin{array}{l}+ \\
-\end{array}$ \\
\hline $\begin{array}{l}03 \\
57\end{array}$ & - & - & + & - & & - \\
\hline 53 & + & & + & & & + \\
\hline 45 & - & - & - & - & & - \\
\hline 43 & + & - & - & + & + & \\
\hline 41 & + & + & + & + & & \\
\hline 39 & & - & - & & & \\
\hline 38 & & & - & & & \\
\hline 35 & - & + & - & & + & - \\
\hline 33 & - & - & - & + & + & \\
\hline 31 & & + & - & + & + & \\
\hline 27 & & & & & + & \\
\hline 25 & + & & + & & - & \\
\hline 22 & & & - & & & \\
\hline 20 & - & - & - & - & + & + \\
\hline 19 & + & - & - & + & - & \\
\hline $15 \cdot 5$ & & & - & & & \\
\hline
\end{tabular}

*High osmolarity was obtained by addition of $\mathrm{NaCl}, \mathrm{KCl}$, sucrose or urea. + or - indicates an increase or a decrease compared to the standard conditions of growth for strain MS11.

† Bacteria were grown under standard conditions. + or - represents proteins which increase or decrease in amount compared to the wild-type strain observed under standard conditions.

\section{DISCUSSION}

In vitro, $N$. gonorrhoeae can only grow under a limited range of environmental conditions. Changes in temperature, $\mathrm{pH}$, salts or amino acid concentrations between values that do not affect growth of enterobacteria are very unfavourable for the growth of $N$. gonorrboeae. However, the gonococcus is able to adapt to several different infection sites where it will be under nutrient limitation or stress conditions. This adaptation is the paradigm of this strictly human pathogen. In the present study, we attempted to analyse the effects of environmental factors on the growth and on the level of pilin, one of the major virulence factors in $N$. gonorrboeae. We have shown that growth is highly sensitive to, and pilin gene expression moderately affected by, a number of environmental factors. We describe a direct cor- relation between growth rate and pilin expression under the majority of the conditions tested (high osmolarity, anaerobiosis, high temperature). Taken together, our results suggest that growth limitation represses the expression of the pilin gene. Our data are in favour of an adaptation behaviour of gonococci to multiple stress signals, as suggested by the numerous conditions that reduce growth and pilin expression simultaneously. The exception of iron starvation, which stimulates pilin gene expression, may be irrelevant since the gonococcus is able to use the lactoferrin and transferrin of the host as an iron source in vivo (Biswas \& Sparling, 1995; Cornelissen \& Sparling, 1996).

It may be advantageous for the gonococcus to vary the level of pilin gene expression according to growth conditions. For instance, a transient reduction in the 


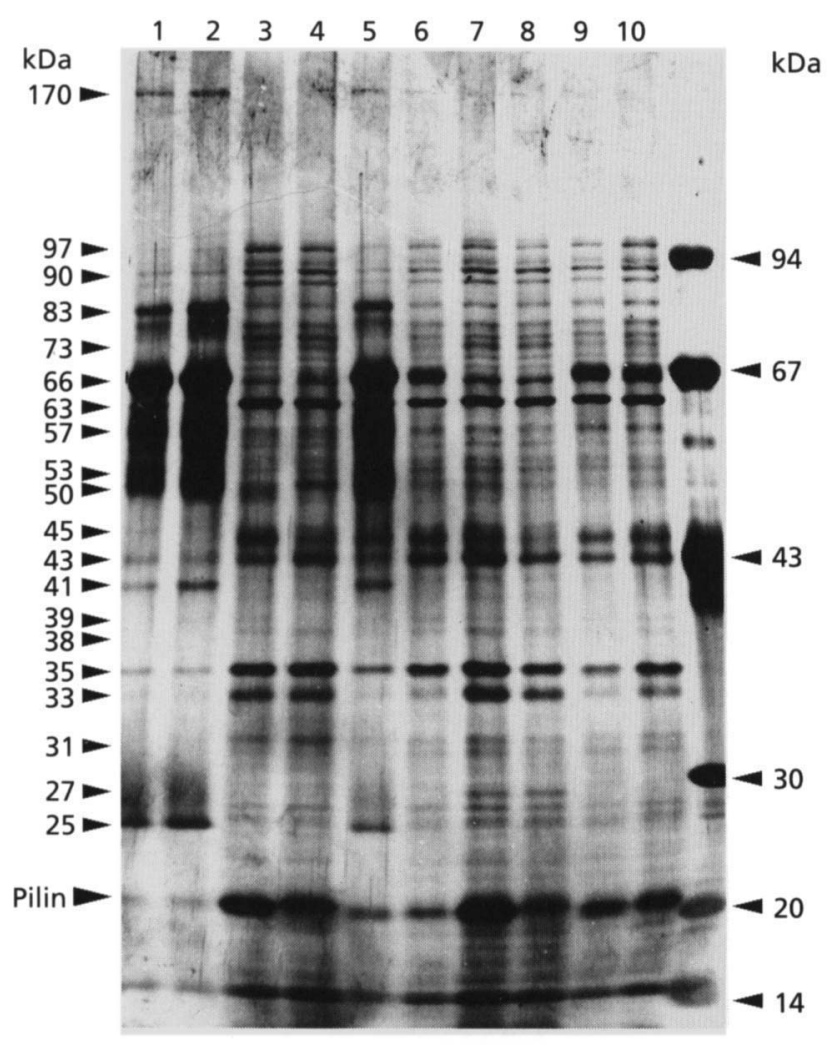

Fig. 4. Silver-nitrate-stained SDS-PAGE gel of total cell extracts of $N$. gonorrhoeae. Bacteria were from anaerobic (lanes 1-5) or aerobic (lanes 6-10) cultures. The strains analysed were MS11pilA ${ }^{+} /$pilAa (lanes 1 and 10), MS11.CAT-2 (lanes 2 and 6), MS11.pilA ${ }^{+} /$pilAk (lanes 3 and 7), MS11.pilA ${ }^{+} /$pilAk.pilBa (lanes 4 and 8) and MS11-A (lanes 5 and 9). Molecular mass markers are shown to the right. Arrowheads on the left indicate the proteins which show variable levels.

pilin level under unfavourable growth conditions might allow the dissemination of bacteria to other sites more favourable for growth, piliation and colonization. Moreover, this modulation of piliation could be useful for the invasion step, as non-piliated gonococci are more efficiently internalized (Makino et al., 1991).

In other pathogenic bacteria, different variations of the growth conditions modulate regulatory genes for virulence factors [ bvgAS in Bordetella pertussis (Manetti et al., 1994; Graeff-Wohlleben et al., 1995; Prugnola et al., 1995), regA in Pseudomonas aeruginosa (Franck et al., 1989; Prince et al., 1991) or toxR in Vibrio cholerae
(Di Rita, 1992)]. This modulation is generally more important for the toxin-encoding genes (10-100-fold) than for the genes implicated in pilus or flagellum biogenesis (2-5-fold). In $N$. gonorrboeae, our studies on the variations in the levels of pilin and other proteins that are affected by environmental changes indicate that the products of pilA and pilB participate in this control. The modification of pilE expression observed in the presence of isoleucine, urea, under anaerobic growth, high osmolarity $(\mathrm{NaCl}$ or $\mathrm{KCl}$ addition) or at $\mathrm{pH} 6$ are lost in the pilBa mutant. These results support the hypothesis that PilB helps transduce different signals to modify pilE expression. In addition, the pilBa mutant showed an increase in the level of several proteins, some of which are related to stress response, such as Gsp63.

Analysis of pilA mutants confirms that their phenotypes depend on the site of insertional inactivation of pilA. The pilAk mutation provokes the loss of pilE modulation in response to environmental changes. These effects may be explained by the absence of interaction between the PilAk and PilB proteins. Indeed, it has been previously shown that PilA and PilB interact to regulate pilE expression (Taha et al., 1991; Taha \& Giorgini, 1995).

The pilA $A^{+} /$pilAa heterodiploid has a reduced level of pilin, which is nevertheless modulated by a majority of the tested conditions in the same manner as the wildtype strain (exceptions are high osmolarity and addition of isoleucine). Moreover, the variations in the protein profiles observed in the wild-type strain are also found in this mutant (data not shown). These results could be explained by the conservation of the interaction between PilAa and PilB but an impaired transcriptional activation of the pilE gene. The two mutants of the pilA gene ( $p i l A a$ and pilAk) thus show different responses to environmental stress and could be representative of mutations affecting different functional domains of PilA. Indeed, we have previously shown that PilA has different functional domains (Taha \& Giorgini, 1995) and that the observed phenotypes in the heterodiploids are not due to the locations of the insertions of the inactivated pilA but rather to the impaired function of PilA (Taha et al., 1988, 1996; Taha, 1993)

PilA/PilB regulatory proteins seem to have pleiotropic effects on the level of several gonococcal proteins. Moreover, this system is involved in the response of gonococci to environmental variations. The identification of the genes encoding these proteins and the analysis of their expression should help to understand

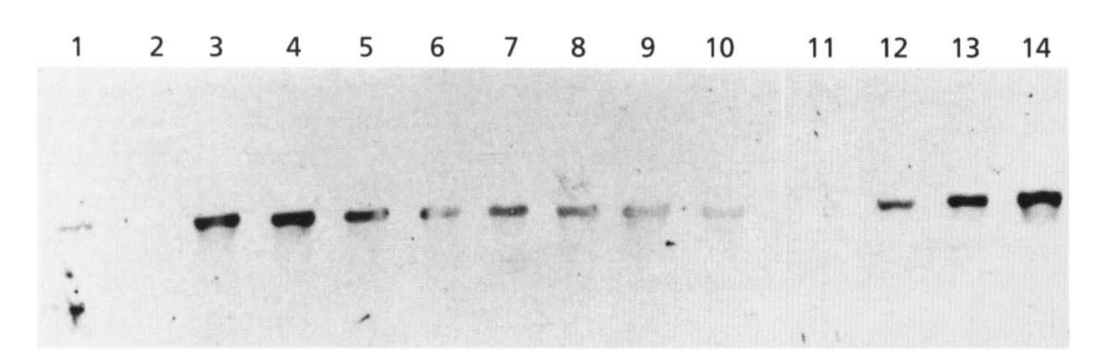

Fig. 5. Western blot analysis of whole-cell extracts of $N$. gonorrhoeae using anti-Gsp63 antibody. Bacteria were grown at $41^{\circ} \mathrm{C}$ (lanes $1-5$ ) or $37^{\circ} \mathrm{C}$ (lanes 6-10) or under anaerobiosis (lanes 11-14). The strains analysed were MS11-A (lanes 1, 6 and 11), MS11.pilA ${ }^{+} /$pilAa (lanes 2, 7 and 12), MS11.pilBa (lanes 3 and 8), MS11.pilA ${ }^{+} /$pilAK (lanes 4, 9 and 13) and MS11.pilA $\%$ /pilAk. pilBa (lanes 5, 10 and 14). 
the adaptation to environmental variations developed by $N$. gonorrhoeae.

\section{ACKNOWLEDGEMENTS}

We would like to thank Yvonne Pannekoek and Bruno Dupuy for the gift of anti Gsp63 and antipilin antibodies, Colin R. Tinsley and Tony Pugsley for critical reading of the manuscript. This work was supported by the INSERM (CRE 930613) and the Institut Pasteur.

\section{REFERENCES}

Biswas, G. D. \& Sparling, P. F. (1995). Characterisation of $l b p A$, the structural gene for a lactoferrin receptor in Neisseria gonorrboeae. Infect Immun 63, 2958-2967.

Cornelissen, C. N. \& Sparling, P. F. (1996). Binding and surface exposure characteristics of the gonococcal transferrin receptor are dependent on both transferrin-binding proteins. $J$ Bacteriol 178, 1437-1444.

Di Rita, V. J. (1992). Coordinate expression of virulence genes by toxR in Vibrio cholerae. Mol Microbiol 6, 451-458.

Dupuy, B., Taha, M.-K., Pugsley, A. P. \& Marchal, C. (1991). Neisseria gonorrhoeae prepilin export studied in Escherichia coli. $J$ Bacteriol 173, 7589-7598.

Franck, D. W., Storey, D. G., Hindahl, M. S. \& Iglewski, B. H. (1989). Differential regulation by iron of $\operatorname{reg} A$ and tox $A$ transcript accumulation in Pseudomonas aeruginosa PA103 and PAO1. J Bacteriol 171, 5304-5313.

Gorby, G. L. \& Schaefer, G. B. (1992). Effect of attachment factors (pili + opa) on Neisseria gonorrhoeae invasion of human fallopian tube tissue in vitro; quantitation by computerized image analysis. Microb Pathog 13, 93-108.

Graeff-Wohlleben, H., Deppisch, H. \& Gross, R. (1995). Global regulatory mechanisms affect virulence gene expression in Bordetella pertussis. Mol Gen Genet 247, 86-94.

Keevil, C. W., Major, N. C., Davies, D. B. \& Robinson, A. (1986). Physiology and virulence determinants of Neisseria gonorrhoeae grown in glucose-, oxygen-, or cystine-limited continuous culture. J Gen Microbiol 132, 3289-3302.

Keevil, C. W., Davies, D. B., Spillane, B. J. \& Mahenthiralingam, E. (1989). Influence of iron-limited and replete continuous culture on the physiology and virulence of Neisseria gonorrboeae. J Gen Microbiol 135, 851-863.

Larribe, M., Taha, M. K. \& Marchal, C. (1991). Control of pilin gene expression in Neisseria gonorrhoeae by environmental factors. In Neisseriae 1990, pp. 441-445. Edited by M. Achtman, P. Kohl, C. Marchal, G. Morelli, A. Seiler \& B. Thiesen. Berlin \& New York: Walter de Gruyter.

Makino, S., van Putten, J. P. M. \& Meyer, T. F. (1991). Phase variation of the opacity outer membrane protein controls invasion by Neisseria gonorrboeae into epithelial cells. EMBO J 10, 1307-1315.

Manetti, R., Arico, R. \& Rappuoli, R. (1994). Mutations in the linker region of BvgS abolish response to environmental signals for the regulation of the virulence factors in Bordetella pertussis. Gene 150, 123-127.

Molesh, I. M., Boxberger, J. \& Meyer, T. F. (1994). Interaction of gonococci with primary epithelial cells from human ureter grown as monolayers and multicell vesicles. Eur J Cell Biol 63, 143.

Pannekoek, Y., Van Putten, J.P. M. \& Dankert, J. (1992).
Identification and molecular analysis of a 63-kilodalton stress protein from Neisseria gonorrboeae. J Bacteriol 174, 6928-6937.

Prince, R. W., Storey, D. G., Vasil, A. I. \& Vasil, M. L. (1991). Regulation of toxA and regA by the Escherichia coli fur gene and identification of a Fur homologue in Pseudomonas aeruginosa PA103 and PAO1. Mol Microbiol 5, 2823-2831.

Prugnola, A., Arico, B., Manetti, R. \& Rappuoli, R. (1995). Response of the bvg operon of Bordetella pertussis to different temperatures and short-term temperature shifts. Microbiology 141, 2529-2534.

Robertson, B. D. \& Meyer, T. F. (1992). Genetic variation in pathogenic bacteria. Trends Genet 8, 422-427.

Rudel, T., Scheuerpflug, I. \& Meyer, T. F. (1995). Neisseria PilC protein identified as type-IV pilus tip-located adhesin. Nature 373, 357-359.

Saikh, K. U., Mitra, S. \& Bhattacharyya, F. K. (1989). Effects of amino acids on colony phenotype of Neisseria gonorrboeae. Infect Immun 27, 1090-1094.

Segal, E., Billyard, E., So, M., Storzbach, S. \& Meyer, T. F. (1985). Role of chromosomal rearrangement in $N$. gonorrboeae pilus phase variation. Cell 40, 293-300.

Seifert, H. S. \& So, M. (1988). Genetic mechanisms of bacterial antigenic variation. Microbiol Rev 52, 327-336.

Shaw, W. V. (1975). Chloramphenicol acetyl transferase from chloramphenicol resistant bacteria. Methods Enzymol 43, 737-755.

Swanson, J., \& Robins, K., Barrera, O., Corwin, D., Boslego, J., Ciak, J., Blake, M. \& Koomey, J. M. (1987). Gonococcal pilin variants in experimental gonorrhoea. $J$ Exp Med 165, 1344-1357. Taha, M. K. (1993). Increased sensitivity of gonococcal pilA mutants to bactericidal activity of normal human serum. Infect Immun 61, 4662-4668.

Taha, M. K. \& Giorgini, D. (1995). Phosphorylation and functional analysis of PilA, a protein involved in the transcriptional regulation of the pilin gene in Neisseria gonorrboeae. Mol Microbiol 15, 667-677.

Taha, M. K., So, M., Seifert, H. S., Billyard, E. \& Marchal, C. (1988). Pilin expression in Neisseria gonorrhoeae is under both positive and negative transcriptional control. EMBO J 7, 4367-4378.

Taha, M. K., Dupuy, B., Saurin, W., So, M. \& Marchal, C. (1991). Control of pilus expression in Neisseria gonorrhoeae as an original system in the two component regulators. Mol Microbiol 5, 137-148.

Taha, M. K., Larribe, M., Dupuy, B., Giorgini, D. \& Marchal, C. (1992). Role of pilA, an essential regulatory gene of Neisseria gonorrhoeae, in stress response. J Bacteriol 174, 5978-5981.

Taha, M. K., Giorgini, D. \& Nassif, X. (1996). The pilA regulatory gene modulates the pilus-mediated adhesion of Neisseria meningitidis by controlling the transcription of pilC1. Mol Microbiol 19, 1073-1084.

Waldbeser, L. S., Ajioka, R. S., Merz, A. J., Puaoi, D., Lin, L., Thomas, T. F. \& So, M. (1994). The opaH locus of Neisseria gonorrhoeae MS11-A is involved in epithelial cell invasion. Mol Microbiol 13, 919-928.

Watt, P. J. \& Ward, M. E. (1980). Adherence of Neisseria gonorrboeae and other Neisseria species to mammalian cells. In Bacterial Adherence, pp. 253-288. Edited by E. H. Beachey. London: Chapman \& Hall.

Received 23 August 1996; revised 2 December 1996; accepted 7 January 1997. 